\title{
CHARACTERIZATIONS OF CERTAIN SPACES OF CONTINUOUS FUNCTIONS
}

\author{
BY \\ MEYER JERISON
}

1. Introduction. Several years ago, there appeared a number of solutions to the problem of characterizing the space of continuous real-valued functions on a compact (bicompact Hausdorff) space using only concepts that are defined in a Banach space. (See [3] $\left({ }^{1}\right),[5]$, [9].) Given a real Banach space, $B$, the problem was to find necessary and sufficient conditions on $B$ in order that there exist a compact space $X$ such that $B$ is equivalent to the space $B(X)$ of continuous functions on $X$, where the usual norm $\|f\|=\sup _{x} \in X|f(x)|$ is used in $B(X)$. The essential feature in the equivalence of any two Banach spaces is the isometry of the spaces, and since the metric properties of a space are determined by the shape of its unit sphere, the conditions appear generally as conditions on the unit sphere of $B$ or of its conjugate space $B^{*}$. One of the necessary conditions given by Arens and Kelley [3] is that $B$ have property $A$ (see definition 2.1). Roughly speaking, the condition is that a collection of faces of the unit sphere of $B$ will have a nonempty intersection unless the collection has faces that are approximately antipodal.

The main result of this paper is a representation of a Banach space with property $A$ as a set of functions. Let $X$ be a compact space and $\sigma$ an involutory homeomorphism of $X$, that is, $\sigma=\sigma^{-1}$. A Banach space has property $A$ if and only if it is equivalent to the space $B_{\sigma}(X)$ of continuous functions on $X$ satisfying the relation $f[\sigma(x)]=-f(x)$ for all $x$ in $X$. This result enables us to omit one of the conditions from the theorem of Arens and Kelley, and also to characterize the Banach space of functions that vanish at infinity on a locally compact space.

2. Property $A$. Throughout this paper, $B$ will denote a real Banach space and $S$ the surface of the unit sphere of $B$. If $C$ is a subset and $b$ an element of $B$, then $d(b, C)=\inf _{c \in C}\|b-c\|$. We will have occasion to use MooreSmith limits, and the terminology of [2] will be used for this purpose. A directed set is a class of elements $\{\alpha\}$ together with a transitive relation $>$ with the property that for every $\alpha_{1}, \alpha_{2}$ there exists $\alpha$ such that $\alpha>\alpha_{1}$ and $\alpha>\alpha_{2}$. A function from a directed set to a collection $M$ will be called a $d i$ rected system of elements of $M$. If $\lambda_{\alpha}$ is a directed system of real numbers, $\lambda_{\alpha} \rightarrow \lambda$ means, for each $\epsilon>0$ there exists $\alpha_{\epsilon}$ such that $\left|\lambda-\lambda_{\alpha}\right|<\epsilon$ for all $\alpha>\alpha_{\epsilon}$.

2.1 Definition. A Banach space satisfying the following condition is

Presented to the International Congress of Mathematicians, September 1, 1950; received by the editors May 13, 1950.

(1) Numbers in brackets refer to the bibliography at the end of the paper. 
said to have property $A$ : If there is no element common to all members of a collection $\Gamma$ of maximal convex subsets of the surface of the unit sphere, then there exist directed systems $C_{\alpha}^{\prime}, C_{\alpha}^{\prime \prime} \in \Gamma$ such that

$$
d\left(b, C_{\alpha}^{\prime}\right)+d\left(b, C_{\alpha}^{\prime \prime}\right) \rightarrow 2
$$

for all $b$ for which $\|b\| \leqq 1$.

Spaces having property $A$ will be studied with the aid of certain concepts introduced by Myers [9]. A $T$-set is a subset, $T$, of a Banach space, $B$, which is maximal with respect to the property that $\sum_{i=1}^{n}\left\|t_{i}\right\|=\left\|\sum_{i=1}^{n} t_{i}\right\|$ whenever $t_{1}, t_{2}, \cdots, t_{n} \in T$. It is easy to see that a subset of $S$ is maximally convex if, and only if, it is the intersection with $S$ of a $T$-set of $B$. Thus, given a $T$-set, $T$, there is a unique maximal convex subset $C$ of $S$, namely, $C=T \cap S$, such that $T=\{\lambda c \mid \lambda \geqq 0, c \in C\}$. It follows from Zorn's lemma that each element of $B$ is contained in at least one $T$-set, or equivalently, each element of $S$ is contained in at least one maximal convex subset of $S$.

With each $T$-set, $T$, of $B$ there is associated a functional $F_{T}$ defined as $F_{T}(b)=\inf _{t \in T}(\|b+t\|-\|t\|)$.

2.2 Lemma. The functional $F_{T}$ has the following properties:

(a) $\left|F_{T}(b)\right| \leqq\|b\|$ for all $b \in B$.

(b) $F_{T}(b)=\|b\|$ if and only if $b \in T$.

(c) If $b \in-T$, then $F_{T}(b)=-\|b\|$.

(d) $F_{T}(\lambda b)=\lambda F_{T}(b)$ for all $\lambda \geqq 0$.

(e) $F_{T}$ is continuous over $B$.

(f) $F_{T_{1}}(b)=F_{T_{2}}(b)$ for all $b \in B$ if and only if $T_{1}=T_{2}$.

(g) $F_{T}$ is linear over $B$ if and only if $F_{T}(b)=-F_{T}(-b)$ for all $b \in B$.

(h) $F_{T}$ is linear if and only if the functional $\|t\|$ defined for $t \in T$ has a unique linear extension of norm 1 over $B$.

The proof of this lemma is contained in [9].

The correspondence between $T$-sets of $B$ and maximal convex subsets of $S$ leads us to expect that property $A$ can be reformulated in terms of $T$-sets. Such a formulation is given in the next definition, although the proof that it is equivalent to property $A$ will not be completed until after the main theorem (3.5) has been proved. We will denote the zero element of $B$ by $\theta$.

2.3 Definition. A Banach space $B$ satisfying the following condition is said to have property $A^{\prime}$ : If $\Delta$ is a collection of $T$-sets of $B$ whose only common element is $\theta$, then there exist directed systems $T_{\alpha}^{\prime}, T_{\alpha}^{\prime \prime} \in \Delta$ such that

$$
F_{\alpha}^{\prime}(b)+F_{\alpha}^{\prime \prime}(b) \rightarrow 0 \quad \text { for all } b \in B .
$$

(We write $F_{\alpha}^{\prime}$ for the functional $F_{T}$ for $T=T_{\alpha}^{\prime}$, and similarly $F_{\alpha}^{\prime \prime}$, in order to simplify the typography.) $A$.

2.4 Lemma. A Banach space has property $\mathcal{A}^{\prime}$ if and only if it has property 
Proof. We prove here only that $A$ implies $\mathcal{A}^{\prime}$.

Let $T$ be a $T$-set of $B$, and $C=T \cap S$. For any $b \in B$ and $t, t_{0} \in T$,

$$
\|b+t\|-\|t\| \geqq\left\|t_{0}+t\right\|-\left\|b-t_{0}\right\|-\|t\|=\left\|t_{0}\right\|-\left\|b-t_{0}\right\| .
$$

It follows that $F_{T}(b) \geqq\left\|t_{0}\right\|-\left\|b-t_{0}\right\|$ for all $t_{0} \in T$, and in particular, for all $t_{0} \in C$. Therefore,

$$
F_{T}(b) \geqq 1-d(b, C)
$$

Also,

$$
F_{T}(b) \leqq \inf _{t_{0}} \in C\left(\left\|b+t_{0}\right\|-\left\|t_{0}\right\|\right)=d(-b, C)-1 .
$$

Suppose $\Delta$ is a collection of $T$-sets of $B$ whose only common element is $\theta$. Let $\Gamma=\{C \mid C=T \cap S, T \in \Delta\}$. Then $\cap\{C \mid C \in \Gamma\}=\varnothing$, and if $B$ has property $\mathcal{A}$, there exist directed systems $C_{\alpha}^{\prime}, C_{\alpha}^{\prime \prime} \in \Gamma$ such that for each $b \in B$ with $\|b\| \leqq 1$, and each $\epsilon>0$, there is an $\alpha_{\epsilon}$ such that

$$
\begin{aligned}
d\left(b, C_{\alpha}^{\prime}\right)+d\left(b, C_{\alpha}^{\prime \prime}\right) & <2+\epsilon, \\
d\left(-b, C_{\alpha}^{\prime}\right)+d\left(-b, C_{\alpha}^{\prime \prime}\right) & <2+\epsilon
\end{aligned}
$$

for all $\alpha>\alpha_{\mathrm{\epsilon}}$. If $T_{\alpha}^{\prime}=\left\{\lambda c \mid c \in C_{\alpha}^{\prime}, \lambda \geqq 0\right\}$ and similarly $T_{\alpha}^{\prime \prime}$, then $T_{\alpha}^{\prime}, T_{\alpha}^{\prime \prime} \in \Delta$, and for all $\alpha>\alpha_{\epsilon}$,

$$
F_{\alpha}^{\prime}(b)+F_{\alpha}^{\prime \prime}(b) \geqq 1-d\left(b, C_{\alpha}^{\prime}\right)+1-d\left(b, C_{\alpha}^{\prime \prime}\right)>-\epsilon
$$

and

$$
F_{\alpha}^{\prime}(b)+F_{\alpha}^{\prime \prime}(b) \leqq d\left(-b, C_{\alpha}^{\prime}\right)-1+d\left(-b, C_{\alpha}^{\prime \prime}\right)-1<\epsilon .
$$

Thus, $F_{\alpha}^{\prime}(b)+F_{\alpha}^{\prime \prime}(b) \rightarrow 0$ for each $b$ satisfying $\|b\| \leqq 1$. By Lemma 2.2(d), this holds for all $b \in B$.

\subsection{Lemma. If $B$ has property $\mathcal{A}^{\prime}$, then each functional $F_{T}$ is linear.}

Proof. If $T$ is a $T$-set of $B$ so also is $-T=\{-t \mid t \in T\}$, and $T \cap-T=\theta\left({ }^{2}\right)$. Since $B$ has property $A^{\prime}$, the collection $\Delta$ consisting of $T$ and $-T$ contains directed systems $T_{\alpha}^{\prime}$ and $T_{\alpha}^{\prime \prime}$ such that $F_{\alpha}^{\prime}(b)+F_{\alpha}^{\prime \prime}(b) \rightarrow 0$ for all $b \in B$. Now, if $t \in T$, then $F_{T}(t)=\|t\|=-F_{-T}(t)$. Since $T$ contains elements different from $\theta$, this implies that for some $\alpha_{0}$, we must have $T_{\alpha}^{\prime}=T$ and $T_{\alpha}^{\prime \prime}=-T$ or $T_{\alpha}^{\prime}=-T$ and $T_{\alpha}^{\prime \prime}=T$ for each $\alpha>\alpha_{0}$. Consequently, $F_{T}(b)=-F_{-T}(b)$ for all $b \in B$. But it follows directly from the definition of the functionals that $F_{-T}(b)=F_{T}(-b)$. Therefore $F_{T}(b)=-F_{T}(-b)$ for all $b \in B$ and so (Lemma $2.2(\mathrm{~g})) F_{T}$ is linear.

(2) We will use the same symbol to denote both the set consisting of a single element and the element itself. 
3. Representation of spaces with property $A$. Let $X$ be a compact space and $\sigma$ an involutory homeomorphism of $X$. The set $B_{\sigma}(X)$ of continuous functions on $X$ satisfying the relation $b[\sigma(x)]=-b(x)$ for all $x$ in $X$ is a complete linear subspace of $B(X)$. The next lemma will be useful in constructing functions that are in $B_{\sigma}(X)$. For example, it is an immediate consequence of the lemma that if $\sigma$ is not the identity map, then $B_{\sigma}(X)$ contains functions that are not identically zero.

3.1 Lemma. If $P$ is a closed set in $X$ and $P \cap \sigma(P)=\varnothing$, then there exists an open set $U \supset P$ such that $U \cap \sigma(U)=\varnothing$.

Proof. Since $X$ is a normal space, there are open sets $U^{\prime}, V^{\prime}$, such that $U^{\prime} \supset P, V^{\prime} \supset \sigma(P)$, and $U^{\prime} \cap V^{\prime}=\varnothing$. The set $U=U^{\prime} \cap \sigma\left(V^{\prime}\right)$ satisfies the requirements of the lemma.

In the next few lemmas, we will extend to $B_{\sigma}(X)$ some known properties of the space $B(X)$.

3.2 Lemma. Let $E$ be a linear subspace of $B(X)$. If $C$ is a maximal convex subset of the surface of the unit sphere of $E$, then there exist $x \in X$ and $\epsilon= \pm 1$ such that $C=\{b \mid b \in E,\|b\|=1, b(x)=\epsilon\}$. Equivalently, if $T$ is a $T$-set of $E$, then $T=\{b \mid b \in E, b(x)=\epsilon\|b\|\}$.

Proof. By hypothesis, $C$ is a convex subset of the surface of the unit sphere of $B(X)$, and it follows from Zorn's lemma that there is a maximal convex subset $C^{\prime}$ containing $C$. For the set $C^{\prime}$, it is known (see [3], for example) that there are $x \in X$ and $\epsilon= \pm 1$ such that $C^{\prime}=\{b \mid b \in B(X),\|b\|=1, b(x)=\epsilon\}$. Since $C$ is maximally convex in the surface of the unit sphere of $E, C=C^{\prime} \cap E$ $=\{b \mid b \in E,\|b\|=1, b(x)=\epsilon\}$. The result for $T$-sets follows from the fact that some $C, T=\{\lambda c \mid \lambda \geqq 0, c \in C\}$.

As distinguished from the situation in $B(X)$, a set $\{b \mid\|b\|=1, b(x)=\epsilon\}$ need not be a maximal convex subset of the surface of the unit sphere of a proper subspace, and if it is maximally convex, $x$ and $\epsilon$ need not be unique.

3.3 Lemma. If $C=\left\{b \mid\|b\|=b\left(x_{0}\right)=1\right\}$ is a maximal convex subset of the surface of the unit sphere of $B_{\sigma}(X)$, then $d(b, C)=1-b\left(x_{0}\right)$ for all $b \in B_{\sigma}(X)$ satisfying $\|b\| \leqq 1$.

Proof. For each $b \in B_{\sigma}(X)$ with $\|b\| \leqq 1$ and $c \in C,\|c-b\| \geqq\left|c\left(x_{0}\right)-b\left(x_{0}\right)\right|$ $=1-b\left(x_{0}\right)$. Hence $d(b, C)=\inf _{c \in C}\|b-c\| \geqq 1-b\left(x_{0}\right)$. To get the inequality reversed, let $b\left(x_{0}\right)=\beta$, and suppose first $\beta>0$. For fixed $\delta, 0<\delta<\beta$, let $U=\{x \in X \mid b(x)>\beta-\delta\} . U$ is an open set containing $x_{0}$, and since $X$ is normal, there is a continuous function $f$ on $X$ such that $f\left(x_{0}\right)=1, f(x)=\beta-\delta$ if $x \notin U$, and $\beta-\delta \leqq f(x) \leqq 1$ for all $x \in X$. Define

$$
\begin{aligned}
c(x) & =f(x) & & \text { for } x \in \bar{U} \text { (the closure of } U) \\
& =b(x) & & \text { for } x \in X-[U \cup \sigma(U)] \\
& =-f[\sigma(x)] & & \text { for } x \in \sigma(\bar{U}) .
\end{aligned}
$$


Since $b(x) \geqq \beta-\delta>0$ when $x \in \bar{U}$, it follows that $\bar{U} \cap \sigma(\bar{U})=\varnothing$. Hence the sets where $c$ is defined overlap only in $\bar{U}-U$ and $\sigma(\bar{U})-\sigma(U)$. On the former, we have $f(x)=\beta-\delta=b(x)$, and on the latter, $-f[\sigma(x)]=-(\beta-\delta)=b(x)$. Thus, $c$ is defined uniquely for each $x \in X$, and since it is continuous on each of a finite number of closed sets whose union is $X$, it is continuous on all of $X$. It is clear that $c \in C$. Now, if $x \in \bar{U},|c(x)-b(x)|=|f(x)-b(x)| \leqq 1-(\beta-\delta)$; if $x \in X-[U \cup \sigma(U)], c(x)-b(x)=0$; if $x \in \sigma(\bar{U})$, then $\sigma(x) \in \bar{U}$, and $\mid c(x)$ $-b(x)|=|-f[\sigma(x)]+b[\sigma(x)] \mid \leqq 1-(\beta-\delta)$. Therefore $\|c-b\| \leqq 1-\beta+\delta$. We can find such a $c \in C$ for each $\delta>0$, and consequently, $d(b, C) \leqq 1-\beta=1$ $-b\left(x_{0}\right)$. Next, suppose $b\left(x_{0}\right)=\beta \leqq 0$. Since $\sigma\left(x_{0}\right) \neq x_{0}$ (for otherwise $C$ would be empty) $x_{0}$ has a neighborhood $V$ such that $V \cap \sigma(V)=\varnothing$, by Lemma 3.1. For given $\delta>0$, let $U=V \cap\{x|| b(x)-\beta \mid<\delta\}$. Then there is a continuous function $f$ defined on $X$ such that $f\left(x_{0}\right)=1, f(x)=0$ for $x \notin U$, and $0 \leqq f(x) \leqq 1$ for all $x \in X$. Let $c(x)=f(x)-f[\sigma(x)]$. For $x \in U,|b(x)-\beta|<\delta$, and since $\beta \leqq 0, \quad|c(x)-b(x)|=|f(x)-b(x)|<1-\beta+\delta ;$ for $x \in \sigma(U), \quad \sigma(x) \in U$ and $|c(x)-b(x)|=|-f[\sigma(x)]+b[\sigma(x)]|<1-\beta+\delta$; for all other $x, c(x)=0$ and $|b(x)| \leqq 1<1-\beta+\delta$. Thus, $\|c-b\|<1-\beta+\delta$. Therefore, $d(b, C) \leqq 1-\beta=1$ $-b\left(x_{0}\right)$ and the proof is complete.

3.4 Lemma. If $T=\left\{b \mid b\left(x_{0}\right)=\|b\|\right\}$ is a T-set of $B_{\sigma}(X)$, then $F_{T}(b)=b\left(x_{0}\right)$ for all $b \in B_{\sigma}(X)$.

Proof. Let $C=\{t \mid t \in T,\|t\|=1\}$. Then $C$ is a maximal convex subset of the surface of the unit sphere, and by the preceding lemma, $d(b, C)=1-b\left(x_{0}\right)$ if $\|b\| \leqq 1$. From (2.1) and (2.2) we have

$$
1-d(b, C) \leqq F_{T}(b) \leqq d(-b, C)-1,
$$

which becomes $b\left(x_{0}\right) \leqq F_{T}(b) \leqq b\left(x_{0}\right)$. Thus, $F_{T}(b)=b\left(x_{0}\right)$ if $\|b\| \leqq 1$, and by Lemma 2.2(d), this holds for all $b \in B_{\sigma}(X)$.

It will be useful to consider the space $B^{*}$ conjugate to $B$, that is, the space of all bounded linear functionals on $B . B^{*}$ is itself a Banach space, with the norm of $F \in B^{*}$ given by $\|F\|=\sup _{|| b|| \leq 1}|F(b)|$. This norm will be used only to single out the unit sphere, $\Sigma=\left\{F \in B^{*} \mid\|F\| \leqq 1\right\}$. When we speak of a topology in $B^{*}$ we will always mean the weak* topology. A neighborhood of $F_{0} \in B^{*}$ in this topology is a set of the form

$$
\left\{F|| F\left(b_{i}\right)-F_{0}\left(b_{i}\right) \mid<\epsilon, b_{i} \in B, \epsilon>0, i=1, \cdots, n\right\} .
$$

An equivalent definition (see [2]) is that a directed system $F_{\alpha}$ converges to $F$ if and only if the system of real numbers $F_{\alpha}(b)$ converges to $F(b)$ for each $b \in B$. In this topology, $\Sigma$ is compact, and for fixed $b, F(b)$ is a continuous function on $\Sigma$.

3.5 Theorem. In order that a Banach space $B$ have property $A$, it is necessary and sufficient that there exist a compact space $X$ and an involutory homeo- 
morphism $\sigma$ of $X$ such that $B$ is equivalent to the space $B_{\sigma}(X)$ of continuous functions on $X$ satisfying the relation $b[\sigma(x)]=-b(x)$ for all $x \in X$.

Proof of sufficiency. Let $\Gamma$ be a collection of maximal convex subsets of the surface of the unit sphere of $B_{\sigma}(X)$ such that $\cap\{C \mid C \in \Gamma\}=\varnothing$. Each $C \in \Gamma$ is of the form given in Lemma 3.2, and since $b[\sigma(x)]=-b(x)$ for every $b \in C$ and $x \in X$, we can always find an $x$ so that $C=\{b \mid b(x)=1=\|b\|]$. Let $P$ be a set of such $x$ 's, one for each $C \in \Gamma$. If $\bar{P} \cap \sigma(\bar{P})=\varnothing$, then there exist an open set $U \supset \bar{P}$ such that $U \cap \sigma(U)=\varnothing$ (Lemma 3.1) and a continuous function $f$ defined on $X$ such that $f(x)=1$ for $x \in \bar{P}, f(x)=0$ for $x \notin U$, and $0 \leqq f(x) \leqq 1$ for all $x \in X$. But then the function $b$ defined by $b(x)=f(x)$ $-f[\sigma(x)]$ is contained in every $C \in \Gamma$, contradicting the assumption on $\Gamma$. Consequently, there is a point $x_{0} \in \bar{P} \cap \sigma(\bar{P})$, or equivalently, both $x_{0}$ and $\sigma\left(x_{0}\right)$ are in $\bar{P}$. This implies the existence of directed systems $x_{\alpha}^{\prime}, x_{\alpha}{ }^{\prime} \in P$ such that $x_{\alpha}^{\prime} \rightarrow x_{0}$ and $x_{\alpha}^{\prime \prime} \rightarrow \sigma\left(x_{0}\right)$. Let $C_{\alpha}^{\prime}, C_{\alpha}^{\prime \prime}$ be the members of $\Gamma$ corresponding to $x_{\alpha}^{\prime}, x_{\alpha}^{\prime \prime}$ respectively. For each $b$ with $\|b\| \leqq 1$, we have $d\left(b, C_{\alpha}^{\prime}\right)$ $=1-b\left(x_{\alpha}^{\prime}\right)$ and $d\left(b, C_{\alpha}^{\prime \prime}\right)=1-b\left(x_{\alpha}^{\prime \prime}\right)$. Therefore, $d\left(b, C_{\alpha}^{\prime}\right) \rightarrow 1-b\left(x_{0}\right)$, $d\left(b, C_{\alpha}^{\prime \prime}\right) \rightarrow 1-b\left[\sigma\left(x_{0}\right)\right]$, and since $b\left(x_{0}\right)=-b\left[\sigma\left(x_{0}\right)\right], d\left(b, C_{\alpha}^{\prime}\right)+d\left(b, C_{\alpha}^{\prime \prime}\right) \rightarrow 2$.

Proof of necessity. If $B$ has property $A$, then by the part of Lemma 2.4 that we have already proved, it has property $\mathcal{A}^{\prime}$. Every functional $F_{T}$ is linear, so that the collection of them is a subset $Y$ of the space $B^{*}$ conjugate to $B$. By Lemma 2.2(a), $Y$ is contained in $\Sigma$, and therefore $\bar{Y}=X$ is a compact set in $\Sigma$. Let $\phi$ be the natural map of $B$ into $B(X)$, that is, if $b \in B$, then $[\phi(b)](x)=x(b)$ for all $x \in X$, where $x(b)$ is the value of the functional $x$ on the element $b$. Since $X \subset \Sigma, \quad\|\phi(b)\|=\sup _{x \in X}|[\phi(b)](x)|=\sup _{x \in X}|x(b)|$ $\leqq\|b\|$. But for each $b \in B$ there is some $T$-set, $T$, containing $b$, so that $F_{T}(b)$ $=\|b\|$. Therefore, $\|\phi(b)\|=\|b\|$, and $\phi$ is an equivalence of $B$ on to a subspace of $B(X)$. The linearity of the functionals $F_{T}$ implies that $F_{T}=-F_{-T}$ for all $T$, so that $Y$, and hence also $X$, contains the negative of each of its members. Let $\sigma: X \rightarrow X$ be the map that takes each element of $X$ into its negative. $\sigma$ is an involutory homeomorphism. For each $x \in X$ and $b \in B,[\sigma(x)](b)$ $=-x(b)$, and therefore $\phi(B) \subset B_{\sigma}(X)$.

We show next that if $P \subset Y$ and $\bar{P} \cap \sigma(\bar{P})=\varnothing$, then there is a $b$ in $B$ such that $\|b\|=1$ and $[\phi(b)](x)=1$ for all $x \in P$. Let $\Delta=\left\{T \mid F_{T} \in P\right\}$. If $\bigcap_{T \in \Delta} T$ contains an element different from $\theta$, then it contains an element $b$ whose norm is 1 . For this element we have $F_{T}(b)=\|b\|=1$ for all $T \in \Delta$, so that $[\phi(b)](x)=1$ for all $x \in P$. On the other hand, if $\bigcap_{T \in \Delta} T=\theta$, then property $\mathcal{A}^{\prime}$ asserts the existence of directed systems $T_{\alpha}^{\prime}, T_{\alpha}^{\prime \prime} \in \Delta$ such that $F_{\alpha}^{\prime}(b)+F_{\alpha}^{\prime \prime}(b) \rightarrow 0$ for all $b \in B$. Since $X$ is compact, the set $\left\{F_{\alpha}^{\prime}\right\}$ has a cluster point $x^{\prime} \in X$. But $\sigma\left(x^{\prime}\right)$ is then a cluster point of the set $\left\{F_{\alpha}^{\prime \prime}\right\}$, and we have $x^{\prime}$ and $\sigma\left(x^{\prime}\right)$ both in $\bar{P}$, or $x^{\prime} \in \bar{P} \cap \sigma(\bar{P})$, contrary to assumption.

It follows from the next lemma that $\phi(B)=B_{\sigma}(X)$, and thus the proof of the theorem is completed. The lemma is of the nature of the generalizations 
of the Weierstrass approximation theorem given by Stone and others (see [6] and [12]) for rings or lattices of continuous functions. Arens and Kelley [3] have essentially the same result for the Banach space $B(X)$.

3.6 Lemma. Let $E$ be a complete linear subspace of $B_{\sigma}(X)$, and $Y$ a dense subset of $X$. If for each set $P \subset Y$ such that $\bar{P} \cap \sigma(\bar{P})=\varnothing, E$ contains an element $b$ such that $\|b\|=1$ and $b(x)=1$ for all $x \in P$, then $E=B_{\sigma}(X)$.

Proof. Suppose $f \in B_{\sigma}(X)$ and $\|f\|=1$. Let $P=\{y \mid y \in Y, f(y) \geqq 1 / 3\}$. Then $f(x) \geqq 1 / 3$ for all $x \in \bar{P}$ and $f(x) \leqq-1 / 3$ for all $x \in \sigma(\bar{P})$, so that $\bar{P} \cap \sigma(\bar{P})=\varnothing$. Let $b_{0}$ be an element of $E$ such that $\left\|b_{0}\right\|=1$ and $b_{0}(y)=1$ for all $y \in P$. Now, $b_{0}(y)=-1$ if $y \in \sigma(P)=\{y \mid y \in Y, f(y) \leqq-1 / 3\}$, and therefore, $\left\|f-(1 / 3) b_{0}\right\|=\sup _{x \in X}\left|f(x)-(1 / 3) b_{0}(x)\right|=\sup _{y \in Y}\left|f(y)-(1 / 3) b_{0}(y)\right|=2 / 3$.

Let $f_{1}(x)=(3 / 2)\left[f(x)-(1 / 3) b_{9}(x)\right]$. Then $f_{1} \in B_{\sigma}(X)$ and $\left\|f_{1}\right\|=1$, so that the same argument will produce $b_{1} \in E$ such that $\left\|b_{1}\right\|=1$ and $\left\|f_{1}-(1 / 3) b_{1}\right\|=2 / 3$. In general, define $f_{i}(x)=(3 / 2)\left[f_{i-1}(x)-(1 / 3) b_{i-1}(x)\right]$ and produce $b_{i} \in E$ such that $\left\|b_{i}\right\|=1$ and $\left\|f_{i}-(1 / 3) b_{i}\right\|=2 / 3$. It is easy to see that for each positive integer $n$,

$$
\left\|f-(1 / 3) \sum_{i=0}^{n-1}(2 / 3)^{i} b_{i}\right\|=(2 / 3)^{n} .
$$

Since $E$ is linear and complete, this implies $f \in E$, and more generally, $E=B_{\sigma}(X)$.

We are now in a position to complete the proof of Lemma 2.4 very easily. The proof of necessity in Theorem 3.5 consisted of showing that if $B$ has property $A^{\prime}$, then $B$ is equivalent to $B_{\sigma}(X)$, and by the sufficiency part of the theorem, this implies $B$ has property $A$.

We consider, next, the possibility of an analogue in $B_{\sigma}(X)$ of the BanachStone theorem $([4 ;$ p. 170] and $[11 ;$ p. 469]) which says that if $B(X)$ and $B\left(X^{\prime}\right)$ are equivalent then $X$ and $X^{\prime}$ are homeomorphic. If $X$ has more than one fixed point under the involution $\sigma$, then the space $X^{\prime}$ obtained by identifying all the fixed points in $X$ and the homeomorphism $\sigma^{\prime}$ of $X^{\prime}$ onto itself induced by $\sigma$ in the obvious manner have the property that $B_{\sigma^{\prime}}\left(X^{\prime}\right)$ is equivalent to $B_{\sigma}(X)$, although $X$ and $X^{\prime}$ need not be homeomorphic. It is not difficult to construct examples of spaces $X$ and $X^{\prime}$ that are not homeomorphic, have the same number of fixed points under their respective involutions $\sigma$ and $\sigma^{\prime}$, and such that $B_{\sigma}(X)$ is equivalent to $B_{\sigma^{\prime}}\left(X^{\prime}\right)$, provided there are at least two fixed points. But the fixed points are the only source of difficulty.

3.7 Theorem. If $X$ and $X^{\prime}$ are compact spaces, $\sigma$ and $\sigma^{\prime}$ are involutory homeomorphisms of $X$ and $X^{\prime}$ respectively, both having either no fixed point or exactly one fixed point, and $B_{\sigma}(X)$ is equivalent to $B_{\sigma^{\prime}}\left(X^{\prime}\right)$, then there exists a homeomorphism $\tau$ of $X$ onto $X^{\prime}$ such that $\sigma=\tau^{-1} \sigma^{\prime} \tau$. 
'This theorem may be proved along the lines of the proofs of the BanachStone theorem in [3] or (in generalized form) in [9]. The following lemma is needed in the latter proof. We will also have use for it later.

3.8 Lemma. If $\sigma\left(x_{0}\right) \neq x_{0}$, then the set $K=\left\{b \mid\|b\|=b\left(x_{0}\right)\right\}$ is a T-set of $B_{\sigma}(X)$. The $T$-set $K$ determines the point $x_{0}$ uniquely.

Proof. It is obvious that $\left\|\sum_{1}^{n} b_{i}\right\|=\sum_{1}^{n}\left\|b_{i}\right\|$ if $b_{i} \in K$. To show that $K$ is maximal with respect to this property, suppose $b_{0} \in K$. Then $\left\|b_{0}\right\|>b_{0}\left(x_{0}\right)$. Let $\delta=\min \left(1,(1 / 2)\left[\left\|b_{0}\right\|-b_{0}\left(x_{0}\right)\right]\right), U^{\prime}=\left\{x \mid b_{0}(x)<\left\|b_{0}\right\|-\delta\right\}, U^{\prime \prime}$ equal a neighborhood of $x_{0}$ such that $U^{\prime \prime} \cap \sigma\left(U^{\prime \prime}\right)=\varnothing$, and $U=U^{\prime} \cap U^{\prime \prime}$. There is a continuous function $f$ on $X$ such that $f\left(x_{0}\right)=1$ and $f(x)=0$ for $x \in U$. Define $b \in B_{\sigma}(X)$ by $b(x)=f(x)-f[\sigma(x)]$. Clearly, $b \in K$. But also, for every $x \in X$, $\left|b(x)+b_{0}(x)\right| \leqq 1+\left\|b_{0}\right\|-\delta=\|b\|+\left\|b_{0}\right\|-\delta$, so that $\left\|b+b_{0}\right\| \leqq\|b\|+\left\|b_{0}\right\|-\delta$ $<\|b\|+\left\|b_{0}\right\|$.

If $K_{1}=\left\{b \mid\|b\|=b\left(x_{1}\right)\right\}$ and $x_{1} \neq x_{0}$, choose a neighborhood $U$ of $x_{0}$ so that $U \cap \sigma(U)=\varnothing$ and $x_{1} \notin U$. Then the function $b$ defined as in the preceding paragraph is in $K$ but not in $K_{1}$. Thus, $K \neq K_{1}$.

4. A characterization of the space $B(X)$. The results of the preceding sections enable us to simplify the characterization of $B(X)$ given by Arens and Kelley [3].

4.1 LEMMA. If $B$ has property $A$, and $e$ is an extreme point and $b_{0}$ any other point of $S$, then there is a maximal convex subset $C$ of $S$ containing $e$ but not $b_{0}$.

Proof. Consider the set $M=\{b \mid b \in S,\|b-e\|<1 / 2\}$. For each $b \in M$, let $C_{b}$ be a maximal convex subset of $S$ containing $b$. Then $d\left(e, C_{b}\right)<1 / 2$ for each $b \in M$, and since $B$ has property $A, \cap_{b \in M} C_{b} \neq \varnothing$. Let $c \in \cap_{b \in M} C_{b}$, and suppose $c \neq e$. If $b_{\lambda}=\lambda e+(1-\lambda) c$, then $\left\|b_{\lambda}\right\|=1$ whenever $0 \leqq \lambda \leqq 1$ because $c \in C_{e}$, and $\left\|b_{\lambda}\right\|>1$ for all $\lambda>1$ because $e$ is an extreme point of $S$ and $c \neq e$. Now, for all $\lambda$ in some neighborhood of $1,\left\|\left(b_{\lambda} /\left\|b_{\lambda}\right\|\right)-e\right\|<1 / 2$. Let $b^{\prime}=b_{\lambda} /\left\|b_{\lambda}\right\|$ for some such $\lambda>1$. Then $b^{\prime} \in M$. But,

$$
\left\|\frac{\left\|b_{\lambda}\right\| b^{\prime}}{\left\|b_{\lambda}\right\|+\lambda-1}+\frac{(\lambda-1) c}{\left\|b_{\lambda}\right\|+\lambda-1}\right\|=\left\|\frac{\lambda e}{\left\|b_{\lambda}\right\|+\lambda-1}\right\|=\frac{\lambda}{\left\|b_{\lambda}\right\|+\lambda-1}<1,
$$

which implies that no convex subset of $S$ contains both $c$ and $b^{\prime}$. This contradicts the assumption that $c \in C_{b^{\prime}}$, and therefore $c=e$. It follows that if $b_{0} \in S, b_{0} \neq e$, then the collection $\left\{C_{b} \mid b \in M\right\}$ already has a member containing $e$ but not $b_{0}$.

4.2 LEMmA. If $B$ has property $A, T_{0}$ is a $T$-set of $B$, and e is an extreme point of $S$, then either $e \in T_{0}$ or $e \in-T_{0}$.

Proof. Let $\Delta$ be the collection of all $T$-sets of $B$ containing $e$. Then $F_{T}(e)=1$ for all $T \in \Delta$. If $T_{0} \cap \bigcap_{T \in \Delta} T=\theta$, then by property $\mathcal{A}^{\prime}, F_{T_{0}}(e)=-1$, which implies $e \in-T_{0}$. Now, it follows from the preceding lemma that 
$\cap\{T \mid T \in \Delta\}=\{\lambda e \mid \lambda \geqq 0\}$. Therefore, if $T_{0}$ meets $\cap\{T \mid T \in \Delta\}$ in any element different from $\theta$, then $e \in T_{0}$.

4.3 Theorem. A Banach space $B$ is equivalent to the space of continuous functions on some compact space if and only if $B$ has property $A$ and the unit sphere of $B$ contains an extreme point.

Proof. If $B$ has property $A$, then it is equivalent to the space $B_{\sigma}(X)$, where $X$ is the closure in $B^{*}$ of the set $Y$ of functionals $F_{T}$, and $\sigma$ is the mapping that takes each functional into its negative. Thus, the elements of $B$ may be considered as functions on $X$. If $e$ is an extreme point of $S$, then $|e(x)|=1$ for all $x \in Y$, by Lemma 4.2, and hence for all $x \in X$. Let $Z=\{x \mid x \in X, e(x)=1\} . Z$ is closed and, therefore, compact. Since $Z \cap \sigma(Z)$ $=\varnothing$ and $Z \cup \sigma(Z)=X$, every continuous function on $Z$ can be extended uniquely to an element of $B_{\sigma}(X)$. It follows that $B(Z)$ is equivalent to $B_{\sigma}(X)$, and so also to $B$.

The converse is contained in Arens and Kelley's theorem.

5. The functions that vanish at infinity on a locally compact space.

5.1 Definition. Let $Z$ be a locally compact (Hausdorff) space. A continuous real-valued function $f$ on $Z$ is said to vanish at infinity if for each $\epsilon>0$ there exists a compact set $Q \subset Z$ such that $|f(z)|<\epsilon$ for $z \notin Q$.

The set of such functions forms a Banach space under the usual definitions, and it will be denoted by $B_{\infty}(Z)$. If $Z$ happens to be compact, then according to the definition, $B_{\infty}(Z)$ coincides with $B(Z)$. Otherwise, $Z$ may be imbedded in a compact space $X$ whose points are those of $Z$ together with one additional point $x_{\infty}$, the "point at infinity." A neighborhood of $x_{\infty}$ is defined as the complement of any compact set in $Z$. Under the natural correspondence, $B_{\infty}(Z)$ is equivalent to the subspace $\left\{b \mid b \in B(X), b\left(x_{\infty}\right)=0\right\}\left({ }^{3}\right)$.

A characterization of the space of continuous functions that vanish at infinity on a locally compact space was given by Abdelhay [1] using properties of rings and lattices. We characterize it here as a Banach space.

5.2 Lemma. In any Banach space $B$, every functional $F_{T}$ that is linear is an extreme point of the unit sphere $\Sigma$ of the conjugate space $B^{*}$.

Proof. By Lemma 2.2 (a) and (b), $\left\|F_{T}\right\|=1$. Suppose $F_{T}=\left(F_{1}+F_{2}\right) / 2$ with $F_{1}, F_{2} \in \Sigma$. Then for every $t \in T, F_{1}(t)+F_{2}(t)=2 F_{T}(t)=2\|t\|$. But $\left|F_{1}(t)\right|$ $\leqq\|t\|$ and $\left|F_{2}(t)\right| \leqq\|t\|$, and therefore $F_{1}(t)=\|t\|=F_{2}(t)$ for all $t \in T$. It follows from Lemma $2.2(\mathrm{~h})$ that $F_{1}=F_{2}=F_{T}$.

5.3 Lemma. If $B$ is any Banach space and $\Omega$ is a subset of $\Sigma$ with the property that for each $b \in B$ there exists $F \in \Omega$ such that $F(b)=\|b\|$, then every extreme point of $\Sigma$ is contained in the closure of $\Omega$.

(3) The compactification of $Z$ by one point and the resulting equivalence of the spaces of functions are valid even if $Z$ is compact. In that case, $x_{\infty}$ is an isolated point of $X$. 
Proof. Let $\Omega^{\prime}$ be the smallest closed convex set containing $\Omega$. Then

$$
\Omega^{\prime}=\left\{F^{\prime} \mid F^{\prime} \in B^{*}, F^{\prime}(b) \leqq \sup _{F} \in \Omega(b) \text { for all } b \in B\right\}\left({ }^{4}\right) .
$$

But it follows from the hypothesis that $\sup _{F \in \Omega} F(b)=\|b\|$, and therefore, $\Sigma \subset \Omega^{\prime}$. Since $\Sigma$ is closed and convex, we have $\Sigma=\Omega^{\prime}$. And by a theorem of Milman [8], every extreme point of $\Omega^{\prime}$ is contained in the closure of $\Omega$.

5.4 Theorem. A Banach space $B$ is equivalent to the space of functions that vanish at infinity on a locally compact space if and only if $B$ has property $A$ and the extreme points of the unit sphere of the conjugate space $B^{*}$ lie in two separated sets that are diametrically opposite each other.

Proof. Let $B$ be equivalent to $B_{\infty}(Z)$. Let $Z^{\prime}$ be a homeomorphic copy of $Z$, and let $Y$ be the space obtained by considering $Z$ and $Z^{\prime}$ as separated sets. $Y$ is locally compact and can be imbedded in a compact space $X=Y \cup x_{\infty}$ as described above. An involution $\sigma$ is defined on $X$ as follows: $\sigma$ maps $Z$ onto $Z^{\prime}$ by the homeomorphic mapping that was assumed to exist, and it maps $Z^{\prime}$ onto $Z$ by the inverse of that mapping; $\sigma\left(x_{\infty}\right)=x_{\infty}$. It is easy to see that $B_{\infty}(Z)$ is equivalent to $B_{\sigma}(X)$, and thus we may identify the space $B$ with $B_{\sigma}(X)$. By Theorem 3.5, $B$ has property $\mathcal{A}$. From Lemmas 3.2 and 3.8 , it follows that there is a one-to-one correspondence between the points of $Y$ and the $T$-sets of $B$ given by $T(y)=\{t \mid t \in B, t(y)=\|t\|\}$. Moreover (Lemma 3.4), $b(y)=F_{T(y)}(b)$ for all $y \in Y$ and $b \in B$. The topology in $B^{*}$ was defined so that the mapping $\Psi: X \rightarrow B^{*}$ given by $\Psi(y)=F_{T(y)}, y \in Y$, and $\Psi\left(x_{\infty}\right)=\theta^{*}$ (the zero of $B^{*}$ ) is a homeomorphism, and by Lemma 5.2, $\Psi(y)$ is an extreme point of $\Sigma$ for each $y \in Y$. Since every element of $B$ is contained in some $T$-set, it follows from Lemma 5.3 that all the extreme points of $\Sigma$ are contained in the closure of $\Psi(Y)$. But $X$ is compact, and therefore $\Psi(X)$ is closed. $\Psi\left(x_{\infty}\right)$ is certainly not an extreme point of $\Sigma$, so that $\Psi(Y)$ coincides with the set of extreme points of $\Sigma$. Now $Y=Z \cup Z^{\prime}$ with $Z$ and $Z^{\prime}$ separated. It follows that $\Psi(Y)=\Psi(Z) \cup \Psi\left(Z^{\prime}\right)$ with $\Psi(Z)$ and $\Psi\left(Z^{\prime}\right)$ separated, and it is clear from the definition of the involution $\sigma$ that $\Psi(Z)$ is diametrically opposite $\Psi\left(Z^{\prime}\right)$.

Conversely, if $B$ has property $A$, then $B$ is equivalent to a space $B_{\sigma}(X)$, where $X$ is a compact subset of $\Sigma$. By Lemma 5.3, $X$ contains all of the extreme points of $\Sigma$. Let $Z$ and $Z^{\prime}$ be separated subsets of $\Sigma$ that are diametrically opposite each other and whose union is the set of extreme points of $\Sigma$. By Lemma 5.2, every functional $F_{T}$ is in $Z \cup Z^{\prime}$. But according to Lemma 3.8, every point of $X$ is a functional $F_{T}$, except $\theta^{*}$, if $\theta^{*}$ happens to be in $X$. Therefore $X-\theta^{*}=Z \cup Z^{\prime}$. Since $X$ is compact and $Z$ and $Z^{\prime}$ are separated, $Z$ and $Z^{\prime}$ are locally compact. It is clear that if $b \in B_{\sigma}(X)$, then, considered as a function on $Z$ only, $b$ vanishes at infinity. Also, if $f$ is a continuous function

(4) A set of this form is called regularly convex (see [7]). According to a result of F. Gantmacher (see [10]), a bounded subset of $B^{*}$ is regularly convex if and only if it is convex and compact in the weak* topology. It follows that the convex closure of $\Omega$ has the given form. 
that vanishes at infinity on $Z$, then there is a unique $b \in B_{\sigma}(X)$ such that $f(z)=b(z)$ for all $z \in Z$; namely, the function $b$ defined as $b(z)=f(z)$ if $z \in Z$, $b\left(z^{\prime}\right)=-f\left[\sigma\left(z^{\prime}\right)\right]$ if $z^{\prime} \in Z^{\prime}$, and if $\theta^{*} \in X, b\left(\theta^{*}\right)=0$. We have, thus, a one-toone correspondence between $B_{\infty}(Z)$ and $B_{\sigma}(X)=B$ which is obviously an equivalence.

\section{BIBLIOGRAPHY}

1. J. Abdelhay, On a theorem of representation, Bull. Amer. Math. Soc. vol. 55 (1949) pp. 408-417.

2. L. Alaoglu, Weak topologies of normed linear spaces, Ann. of Math. vol. 41 (1940) pp. 252-267.

3. R. F. Arens and J. L. Kelley, Characterizations of the space of continuous functions over a compact Hausdorff space, Trans. Amer. Math. Soc. vol. 62 (1947) pp. 499-508.

4. S. Banach, Théorie des opérations linéaires, Warsaw, 1932.

5. J. A. Clarkson, A characterization of C-spaces, Ann. of Math. vol. 48 (1947) pp. 845-850.

6. E. Hewitt, Generalizations of the Weierstrass approximation theorem, Duke Math. J. vol. 14 (1947) pp. 419-427.

7. M. Krein and V. Šmulian, On regularly convex sets in the space conjugate to a Banach space, Ann. of Math. vol. 41 (1940) pp. 556-583.

8. D. Milman, Characteristics of extremal points of regularly convex sets (in Russian), C. R. (Doklady) Acad. Sci. URSS. vol. 57 (1947) pp. 119-122.

9. S. B. Myers, Banach spaces of continuous functions, Ann. of Math. vol. 49 (1948) pp. 132-140.

10. V. Smulian, Sur les topologies différentes dans l'espace de Banach, C.R. (Doklady) Acad. Sci. URSS. vol. 23 (1939) pp. 331-334.

11. M. H. Stone, Applications of the theory of Boolean rings to general topology, Trans. Amer. Math. Soc. vol. 41 (1937) pp. 375-481.

12. - The generalized Weierstrass approximation theorem, Mathematics Magazine vol. 21 (1947) pp. 167-183, 237-254.

UNIVERSITY OF ILLINOIS, URBANA, ILL. 